\title{
Mathematical and physical meaning of the Bell inequalities.
}

\author{
Emilio Santos \\ Departamento de Física. Universidad de Cantabria. Santander. Spain
}

March, 27, 2016

\begin{abstract}
It is shown that the Bell inequalities are closely related to the triangle inequalities involving distance functions amongst pairs of random variables with values $\{0,1\}$. A hidden variables model may be defined as a mapping between a set of quantum projection operators and a set of random variables. The model is noncontextual if there is a joint probability distribution. The Bell inequalities are necessary conditions for its existence. The inequalities are most relevant when measurements are performed at space-like separation, thus showing a conflict between quantum mechanics and local realism (Bell's theorem). The relations of the Bell inequalities with contextuality, Kochen-Specker theorem, and quantum entanglement are briefly discussed.

Key words: Bell inequalities, triangle inequalities in probability theory, Bell theorem, Kochen-Specker theorem
\end{abstract}

\section{Introduction}

More than fifty years have elapsed since John Bell derived his celebrated inequalities [1], 22. These inequalities are fulfilled by all classical theories but are violated by quantum predictions for some quantum states. Thus they prove the impossibility of supplementing quantum mechanics with local hidden variables, a result known as Bell's theorem. This is popularly stated saying that local realism is incompatible with quantum mechanics. Bell's work had a relevant impact on the foundations of quantum mechanics. In 
particular, it has produced a renewed interest in the study of quantum entanglement. It has also led to increasingly refined experiments and it has been a stimulus for the development of quantum information theory. In the last year Bell's theorem has received new attention due to the fact that loophole-free tests of the inequalities have been performed for the first time [3], 4], [5].

In a wide sense the name "Bell inequality" is applied to any inequality that is a necessary condition for the existence of local realisitc (or local hidden variables models). For a recent review see[6]. In this paper I will be concerned with a different, although related, definition of Bell inequalities, namely quadrilateral inequalities involving distances defined in (classical) probability theory for sets of dichotomic random variables. The fulfillement, or not, of the inequalities for similar distances defined for expectation values of projection operators in a Hilbert space allows us to know whether some subsets of operators may be represented, or not, in terms of (classical) probability theory. This connection is stressed in our derivation of the inequalities in section 2.

Actually, those properties are related to the distributivity of classical logic. Indeed, as Birkhoff and von Neumann pointed out[7], the essential difference beween classical and quantum physics appears already at the level of the logic (or propositional calculus), which is distributive in the classical case but not in the quantum case. This relation of the Bell inequalities with the distributivity of the logic has been discussed since long ago [8], [9], [10], [11], [12], [13].

The physical implications of the Bell inequalities will be briefly discussed in section 3. However, I shall touch but slightly on the experimental tests of the inequalities and not at all on the applications to quantum information theory. The main purpose of the section will be to comment on the relevance of the inequalities for the interpretation of quantum mechanics, in particular the question whether a realistic interpretation is possible. There are a lot of papers and many books concerned with this question, that will not be commented here (see, e.g. [14], [15], [16], [17], [18]). The subject is still controversial[19].

\section{Mathematical content}

From the mathematical point of view the Bell inequalities are necessary conditions for the existence of a joint probability distribution associated to a 
set of projection operators (projectors) and a density operator on a Hilbert space. A relevant result is that, if all projectors of the set commute with each other, then a joint probability distribution exists and all Bell inequalities hold true for any density operator. Although this section deals with mathematical properties, and therefore it is independent of any physical theory, I will use the widely known language of quantum mechanics. In particular in this section I shall name "observable" any self-adjoint operator in the Hilbert space.

\subsection{Probability distribution of an observable}

For any pair $\{\hat{A}, \hat{\rho}\}$ of an observable and a density operator it is possible to define the probability density, $f(a)$, of a random variable, $a$, associated with the observable. In fact the standard rule about the expectation value, $\left\langle\hat{A}^{n}\right\rangle$, of the n'th power of an observable $\hat{A}$ in the state given by the density operator $\hat{\rho}$ allows getting the characteristic function, $C(\zeta)$, of the associated probability distribution, that is

$$
\left\langle\hat{A}^{n}\right\rangle=\operatorname{Tr}\left[\hat{A}^{n} \hat{\rho}\right] \Rightarrow C(\zeta)=\operatorname{Tr}[\exp (i \zeta \hat{A}) \hat{\rho}]
$$

where $\operatorname{Tr}(\hat{x})$ means the trace of the operator $\hat{x}$. The probability density is obtained by Fourier transform, that is

$$
\begin{aligned}
f(a) & =\frac{1}{2 \pi} \int d \zeta \exp (-i \zeta a) C(\zeta) \\
& =\frac{1}{2 \pi} \int d \zeta \exp (-i \zeta a) \operatorname{Tr}[\exp (i \zeta \hat{A}) \hat{\rho}] .
\end{aligned}
$$

Example 1: Observable $\hat{A}$ having discrete nondegenerate spectrum and $\hat{\rho}$ being a pure state, that is

$$
\hat{A}=\sum_{j} a_{j}\left|\psi_{j}\right\rangle\left\langle\psi_{j}|, \hat{\rho}=| \phi\right\rangle\langle\phi|
$$


Assuming that the eigenfunctions $\left\{\left|\psi_{j}\right\rangle\right\}$ form an orthonormal basis we have

$$
\begin{aligned}
\exp (i \zeta \hat{A}) & =\sum_{k} \frac{\left(i \zeta \sum_{j} a_{j}\left|\psi_{j}\right\rangle\left\langle\psi_{j}\right|\right)^{k}}{k !} \\
& =\sum_{k} \frac{(i \zeta)^{k}}{k !} \sum_{j}\left(a_{j}\right)^{k}\left|\psi_{j}\right\rangle\left\langle\psi_{j}\left|=\sum_{j} \exp \left(i \zeta a_{j}\right)\right| \psi_{j}\right\rangle\left\langle\psi_{j}\right|
\end{aligned}
$$

whence, taking eq.(1) into account, the probability distribution is

$$
\begin{aligned}
f(a) & =\frac{1}{2 \pi} \sum_{j} \int d \zeta \exp \left(-i \zeta a+i \zeta a_{j}\right)\left\langle\phi \mid \psi_{j}\right\rangle\left\langle\psi_{j} \mid \phi\right\rangle \\
& =\sum_{j} \delta\left(a-a_{j}\right) p_{j}, p_{j} \equiv\left|\left\langle\phi \mid \psi_{j}\right\rangle\right|^{2} .
\end{aligned}
$$

This means that the distribution is discrete and it associates the probability $\left|\left\langle\phi \mid \psi_{j}\right\rangle\right|^{2}$ to the eigenvalue $a_{j}$.

Example 2. $\hat{A}$ is a projector and $\hat{\rho}$ arbitrary (not necessarily representing a pure state). $\hat{A}$ fulfils

$$
\hat{A}=\hat{A}^{2} \Rightarrow \hat{A}^{n}=\hat{A},
$$

for any natural number $n$. Then we have

$$
\begin{aligned}
f(a) & =\frac{1}{2 \pi} \sum_{j} \int d \zeta \exp (-i \zeta a) \operatorname{Tr}[\hat{\rho} \exp (i \zeta \hat{A})] \\
& =\frac{1}{2 \pi} \sum_{j} \int d \zeta \exp (-i \zeta a+i \zeta) \operatorname{Tr}(\hat{\rho} \hat{A})=\delta(a-1) \operatorname{Tr}(\hat{\rho} \hat{A}),
\end{aligned}
$$

which means that $\operatorname{Tr}(\hat{\rho} \hat{A})=p(a)$ is the probability that the projector takes the value 1 .

\subsection{Joint distribution for several observables}

Generalizing eq.(1) we might define the joint probability distribution of two observables, $f(a, b)$, as follows

$$
f(a, b)=\frac{1}{4 \pi^{2}} \int d \zeta \int d \chi \exp (-i \zeta a-i \chi b) \operatorname{Tr}[\exp (i \zeta \hat{A}+i \chi \hat{B}) \hat{\rho}] .
$$


If the observables $\hat{A}$ and $\hat{B}$ commute with each other, the function $f(a, b)$ is indeed the desired joint distribution and it has the properties of a classical joint distribution, as shown in the following. In fact in this case there is a complete set of orthonormal simultaneous eigenvectors of the two observables, that we will label $\left\{\left|\phi_{j}\right\rangle\right\}$ (assuming for simpliticity that the set is discrete). Thus we may use the resolution of the identity operator $\hat{I}$ in terms of these eigenvectors in order to define the trace, i. e.

$$
\hat{I}=\sum_{j}\left|\phi_{j}\right\rangle\left\langle\phi_{j}\right|, \operatorname{Tr} \hat{M}=\left\langle\phi_{j}|\hat{M}| \phi_{j}\right\rangle .
$$

Hence eq.(2) becomes, after some algebra,

$$
f(a, b)=\sum_{j} P_{j} \rho_{a j}(a) \rho_{b j}(b), P_{j}=\left\langle\phi_{j}|\hat{\rho}| \phi_{j}\right\rangle
$$

where $\rho_{a j}(a)$ is the probability distribution of $a$ in the state $j$, as given by eq.(11) . Eq.(3) has the same form as a classical joint probability distribution written in terms of the probability distributions of the individual variables $a$ and $b$. In particular if the sum in $j$ contains only one term, the distributions $\rho_{a j}(a)$ and $\rho_{b j}(b)$ are uncorrelated.

In sharp contrast if $\hat{A}$ and $\hat{B}$ do not commute the function $f(a, b)$ defined by eq.(2) may not be semidefinite positive. For instance in a system consisting of a single particle (without spin) in one dimension, the position and momentum observables, $\hat{x}$ and $\hat{p}$, do not commute and eq.(2) leads to

$$
W(x, p)=\frac{1}{4 \pi^{2}} \int d \zeta \int d \chi \exp (-i \zeta x-i \chi p) \operatorname{Tr}[\exp (i \zeta \hat{x}+i \chi \hat{p}) \hat{\rho}],
$$

which is the Wigner function associated to the state $\hat{\rho}$. As is well known the Wigner function is not always positive. We might try other choices for the ordering of the operators $\hat{x}$ and $\hat{p}$, but none fully satisfactory has been found. Thus the question arises, is it always possible to find a joint (positive semidefinite) probability distribution for several observables and a given density operator?. The answer is affirmative if all observables commute, but negative in general. In order to prove that assertion let us start studying joint probability distributions in the mathematical theory of probability. 


\subsection{Joint distribution for several dichotomic random variables}

What I will present in the following is well kown for any mathematical measure, in particular a probability distribution, defined on a set. In mathematics a measure on a set is a mapping of subsets on nonnegative numbers, with some topological restrictions on the subsets if the set is not finite. But for our purposes it is enough to study probability distributions on a finite set $\left\{a_{j}, j=1,2, \ldots n\right\}$ of random variables having values $\{0,1\}$.

The question that I will try to answer is the following. Given the probabilities $\left\{p_{1}\left(a_{j}\right)\right\}$ that $a_{j}=1$, and the probabilities $\left\{p_{2}\left(a_{j} a_{k}\right)\right\}$ that $a_{j}=a_{k}=1$ for all $j, k$, we want to know whether these probabilities are the marginals of some joint probability distribution for all the variables. A well known result of probability theory is the following lemma: A necessary condition for the existence of a joint probability distribution is that all triangle inequalities of the form

$$
d\left(a_{j}, a_{k}\right)+d\left(a_{k}, a_{l}\right) \geq d\left(a_{j}, a_{l}\right)
$$

hold true. The "distance function" $d\left(a_{j}, a_{k}\right)$ is defined by

$$
d\left(a_{j}, a_{k}\right)=p\left(a_{j} \bar{a}_{k}\right)+p\left(\bar{a}_{j} a_{k}\right)=p_{1}\left(a_{j}\right)+p_{1}\left(a_{k}\right)-2 p_{2}\left(a_{j} a_{k}\right),
$$

where $\bar{a}_{j}=1-a_{j}$ is the variable that takes the value $1(0)$ when $a_{j}$ takes the value $0(1)$. The distance function fulfils

$$
0 \leq d\left(a_{j}, a_{k}\right) \leq 1, d\left(a_{j}, a_{j}\right)=0, d\left(a_{j}, \bar{a}_{j}\right)=1,
$$

We see that the distance is zero when the two variables are maximally correlated (they might be considered the same variable) and it is unity if they are maximally anticorrelated. The proof of the lemma is easy. We consider 3 random varibles $\{a, b, c\}$ with values $\{0,1\}$ and use a Venn diagram where the random variables are represented by circles and the areas are proportional to the probabilities. Thus it is trivial to check that

$$
p(a \bar{b})+p(\bar{a} b)+p(a \bar{c})+p(\bar{a} c) \geq p(b \bar{c})+p(\bar{b} c)
$$

which leads to a triangle inequality similar to eq.(4).

In the following I show that the triangle inequalities eq.(44) are closely related to the Bell inequalities[1]. 


\subsection{Random variables representations}

Let us consider a finite set, $P \equiv\left\{\hat{a}_{j}\right\}$, of projectors (with eigenvalues 1 and 0 ) and a density operator $\hat{\rho}$. A set $R \equiv\left\{a_{j}\right\}$ of random variables with values $\{0,1\}$ is here defined as a "random variables representation" (RVR) of the pair $\{P, \hat{\rho}\}$ if there is an one to one mappping $P \rightarrow R$ such that, for any subset of $P$ involving only projectors that commute with each other, the following equality holds true

$$
\operatorname{Tr}\left[\hat{\rho}\left(\hat{a}_{j} \hat{a}_{k} \ldots \hat{a}_{l}\right)\right]=p\left(a_{j} a_{k} \ldots a_{l}\right),
$$

where $p\left(a_{j}, a_{k} \ldots a_{l}\right)$ is the probability that all variables $\left\{a_{j}, a_{k} \ldots a_{l}\right\}$ take the value 1 . We assume that both sets $P$ and $R$ are complete in the sense that if $\hat{a}_{j} \in P$ also $\hat{I}-\hat{a}_{j} \in P, \hat{I}$ being the identity operator. Hence if $a_{j} \in R$ also $\bar{a}_{j} \in R$.

The quantities on the right side of eq.(17) have all the properties of probabilities. In fact they are positive or zero and the marginals are consistent with the mapping $P \rightarrow R$, that is

$$
\begin{aligned}
p\left(a_{k} \ldots a_{l}\right) & =p\left(a_{j} a_{k} \ldots a_{l}\right)+p\left(\bar{a}_{j} a_{k} \ldots a_{l}\right) \\
& =\operatorname{Tr}\left[\hat{\rho}\left(\hat{a}_{j} \hat{a}_{k} \ldots \hat{a}_{l}\right)\right]+\operatorname{Tr}\left[\hat{\rho}\left(\left(\hat{I}-\hat{a}_{j}\right) \hat{a}_{k} \ldots \hat{a}_{l}\right)\right] \\
& =\operatorname{Tr}\left[\hat{\rho}\left(\hat{a}_{k} \ldots \hat{a}_{l}\right)\right] .
\end{aligned}
$$

In conclusion a "random variables representation" exists for any pair $\{P, \hat{\rho}\}$.

Example: Let us consider four projectors $\left\{\hat{a}_{1}, \hat{b}_{1}, \hat{a}_{2}, \hat{b}_{2}\right\}$ such that $\hat{a}_{j}$ commutes with $\hat{b}_{k}$ for any $j, k \in\{1,2\}$, but neither $\hat{a}_{1}$ commutes with $\hat{a}_{2}$ nor $\hat{b}_{1}$ with $\hat{b}_{2}$. Thus eqs.(7) allow defining 24 probabilities, namely

$$
p\left(a_{j}\right), p\left(b_{k}\right), p\left(\bar{a}_{j}\right), p\left(\bar{b}_{k}\right), p\left(a_{j} b_{k}\right), p\left(\bar{a}_{j} b_{k}\right), p\left(a_{j} \bar{b}_{k}\right), p\left(\bar{a}_{j} \bar{b}_{k}\right) .
$$

But there are many probabilities which are not defined by eqs.(17), namely those involving two or more noncommuting operators. For instance the following probabilities cannot be derived that way

$$
p\left(a_{1} a_{2}\right), p\left(b_{1} b_{2}\right), p\left(a_{1} a_{2} b_{k}\right), p\left(b_{1} b_{2} a_{j}\right), p\left(a_{1} a_{2} b_{1} b_{2}\right) .
$$

There are two different kinds of random variables representation. A RVR of the pair $\{P, \hat{\rho}\}$ is "complete" if there is a joint probability distribution 
for all random variables of $R$ such that the probabilities given by eqs.(77) are marginals of the joint distribution. It is incomplete if such a joint probability does not exist.

In the above example a complete RVR would require the existence of the 16 probabilities

$$
p\left(a_{1} a_{2} b_{1} b_{2}\right), p\left(a_{1} a_{2} b_{1} \bar{b}_{2}\right), p\left(a_{1} a_{2} \bar{b}_{1} b_{2}\right), \ldots p\left(\bar{a}_{1} \bar{a}_{2} \bar{b}_{1} \bar{b}_{2}\right),
$$

whence all other probabilities could be obtained as marginals. However some of these could not be derived from eqs.(7), for instance this would be the case for the probabilities eqs.(9) in our example. On the other hand it may be that probabilities eqs.(10) do not exist with the condition that all probabilities derivable from eqs.(17) are marginals of these eqs.(10). In this case the RVR will not be complete. The complete and incomplete RVR correspond to the socalled noncontextual and contextual hidden variables theories in quantum mechanics, respectivley, as will be clarified in the next section.

It is not difficult to generalize RVR to observables not necessarily projectors. Actually any set of observables may be written (or accuratelly approximated) in terms of projectors.

\subsection{Quadrilateral inequalities as tests of completeness}

An interesting task is to find conditions that allow to discover whether the RVR of a pair $\{P, \hat{\rho}\}$ is complete. In view of the subsection 1.3 a necessary condition would be that all triangle inequalities involving probabilities defined by eq.(7) are fulfilled. However this criterion is useless. In fact for any 3 commuting projectors the triangle inequality holds true either if the RVR is complete or not. On the other hand if two projectors do not commute their distance function cannot be defined because the joint probability is not given by eq.(77). In this cases one of the sides of the triangle inequality is unknown.

The solution to the problem is to consider quadrilateral inequalities as follows. If the RVR is complete, then a joint probability is defined for any 3 random variables of the model. As a consequence, choosing any four variables $\left\{a_{1}, a_{2}, b_{1}, b_{2}\right\}$ the following two triangle inequalities hold true

$$
d\left(a_{1}, b_{1}\right) \leq d\left(a_{1}, a_{2}\right)+d\left(a_{2}, b_{1}\right), d\left(a_{1}, a_{2}\right) \leq d\left(a_{1}, b_{2}\right)+d\left(b_{2}, a_{2}\right),
$$

although it may be that some of the distances cannot be obtained via eqs.(7) (but they might be obtained as marginals of the assumed joint probability 
distribution as the RVR is complete). The addition of these two inequalities gives the quadrilateral inequality

$$
d\left(a_{1}, b_{1}\right) \leq d\left(a_{1}, b_{2}\right)+d\left(b_{2}, a_{2}\right)+d\left(a_{2}, b_{1}\right),
$$

which is usefull because all four distances may be obtained via eqs.(17). As a consequence a necessary condition for a RVR to be complete is that all quadrilateral inequalities like eq.(11) hold true. The inequalities are not trivial if some of the projectors associated with the variables do not commute. For instance, in our example above the projectors $\hat{a}_{1}$ and $\hat{a}_{2}$ do not commute.

The quadrilateral inequalities are closely related to the most typical Bell inequalities [1], 2]. In fact, taking eq.(5) into account, (11) may be written in the form

$$
p\left(a_{1}\right)+p\left(b_{1}\right) \geq p\left(a_{1}, b_{1}\right)+p\left(a_{2}, b_{1}\right)+p\left(a_{1}, b_{2}\right)-p\left(a_{2}, b_{2}\right),
$$

which is an inequality derived by Clauser and Horne[20] in 1974 from different (but equivalent) assumptions as those used here. Most popular is the inequality derived in 1969 by Clauser, Horne, Shimony and Holt (CHSH) [21] which is acually equivalent to eq.(12). In fact, let us consider dichotomic variables $\left\{A_{j}, B_{k}\right\}$ with values $\{-1,1\}$, related to the variables $\left\{a_{j}, b_{k}\right\}$, with values $\{0,1\}$, by means of

$$
a_{j}=\frac{1}{2}\left(A_{j}+1\right), b_{k}=\frac{1}{2}\left(B_{k}+1\right) .
$$

Now for a random variable $a$ with values $\{0,1\}$ the probability $p(a)$ of taking the value 1 is identical to the expectation value $\langle a\rangle$. Thus the inequality eq.(12) may be rewritten

$$
\left\langle a_{1}\right\rangle+\left\langle b_{1}\right\rangle \geq\left\langle a_{1} b_{1}\right\rangle+\left\langle a_{2} b_{1}\right\rangle+\left\langle a_{1} b_{2}\right\rangle-\left\langle a_{2} b_{2}\right\rangle,
$$

which, taking eq.(13) into account, leads to

$$
\begin{aligned}
\frac{1}{2}\left\langle A_{1}+1\right\rangle+\frac{1}{2}\left\langle B_{1}+1\right\rangle \geq & \frac{1}{4}\left\langle\left(A_{1}+1\right)\left(B_{1}+1\right)\right\rangle+\frac{1}{4}\left\langle\left(A_{2}+1\right)\left(B_{1}+1\right)\right\rangle \\
& +\frac{1}{4}\left\langle\left(A_{1}+1\right)\left(B_{2}+1\right)\right\rangle-\frac{1}{4}\left\langle\left(A_{2}+1\right)\left(B_{2}+1\right)\right\rangle .
\end{aligned}
$$

Hence simple algebra gives

$$
2 \geq\left\langle A_{1} B_{1}\right\rangle+\left\langle A_{2} B_{1}\right\rangle+\left\langle A_{1} B_{2}\right\rangle-\left\langle A_{2} B_{2}\right\rangle,
$$


which is the CHSH inequality[21].

I shall point out that in papers dealing with the CHSH inequality it is frequent to call "correlation" the expectation value of a product of observables like $\langle A B\rangle$. The name does not agree with the standard one in mathematical statistics, where the correlation between two random variables, $A$ and $B$, is usually defined by the dimensionless quantity

$$
\operatorname{Corr}(A, B) \equiv \frac{\langle A B\rangle-\langle A\rangle\langle B\rangle}{\sqrt{\left\langle A^{2}\right\rangle-\langle A\rangle^{2}} \sqrt{\left\langle B^{2}\right\rangle-\langle B\rangle^{2}}} .
$$

An inequality similar to CHSH with the correlation eq. (15) substituted for the expectation of the product, $\langle A B\rangle$, may be violated by classical (hidden variables) models. This fact has lead some authors to misunderstand, and criticize, Bell's work.

\section{Physical implications}

The physical meaning of the Bell inequalities has been widely discussed and a detailed survey is beyond the scope of this paper. Here I will give a summary of the fundamental ideas. The interested reader may consult the review by Brunner et al.[6]. At a difference with the previous (mathematical) section, here it is necessary to distinguish between an "observable" of a physical system, say $A$, and the "operator" associated to it in the Hilbert space formalism of quantum mechanics, say $\hat{A}$.

\subsection{Observables and elements of reality}

\subsubsection{From EPR to hidden variables}

The starting point of Bell's work was the celebrated article by Einstein, Podolsky and Rosen (EPR) 22]. Indeed the title of the pioneer Bell publication made reference to that paper[1]. EPR article begins with what may be seen as a definition of epistemological realism, that is the conditions for a realistic interpretation of physics: "Any serious consideration of a physical theory must take into account the distinction between the objective reality, which is independent of any theory, and the physical concepts with which the theory operates. These concepts are intended to correspond with the objective reality, and by means of these concepts we picture this reality to 
ourselves" 22. Then the authors present a state of a pair of particles entangled in positions and momenta. They stress the quantum prediction that, even if the particles are well separated, a measurement of position (momentum) on the first particle allows an instantaneous knowledge of the position (momentum) of the second one. Thus we should attach to the second particle different quantum states without in any way making a physical action on it. The authors conclude that either there is an action at a distance (nonlocality) or quantum mechanics associates two different quantum wavefunctions to the same actual state of the second particle. Thus the EPR paper proves that quantum mechanics is either nonlocal or incomplete, and the authors considered the second alternative more plausible. Bell showed that if quantum mechanics is incomplete (and it admits hidden variables) it is also incompatible with local realism (Bell's theorem). Therefore the question of completeness is irrelevant for the question of compatibility. In a soon reply to EPR, Bohr supported completeness and a kind of wholeness of quantum mechanics that implies nonlocality[23]. Thus Bell's theorem is currently interpreted as a vindication of Bohr against Einstein.

Many physicists do not accept EPR realism, they believe that "pictures of the objective reality" are irrelevant for physics where only the agreement of the theoretical predictions with the empirical evidence is required. However other physicists search for a clear picture of the reality, which is not offered by the quantum formalism. A standard approach to a picture is the introduction of the socalled "hidden variables theories", that might reduce quantum theory to a stochastic theory with classical flavour. The main achievement of Bell's work was to put constraints on the hidden variables models that are possible, in particular proving the impossibility of local models.

\subsubsection{Measurement in macro and microsystems}

Essential in any branch of natural science are the experiments, consisting of just observations (as in astronomy) or preparations followed by measurements (as in laboratory work). Thus it is worth to study the differences between the experiments dealing with macroscopic and microscopic systems.

The most popular difference is of course the fact the some quantities may possess values only within a discrete set in microphysics. Hence the name quantum introduced by Planck. However more important for the hidden variables problem is the empirical fact that macroscopic systems are perturbed but slightly by measurements, whilst in the microscopic domain the state 
of the system is usally dramatically changed. One consequence is that the uncertainties in the results of the measurements in the macroscopic domain are usually small as compared with the result obtained. Thus it is assumed that the perturbation may be reduced indefinitely and therefore ignored in the formulation of classical theories, dealing with macroscopic systems. The logical consequence is to attach the observable quantities to the physical systems themselves, whence the belief that measurements just revail intrinsic properties of the system under study. Thus classical theories dispense with a theory of measurement. In fact the process of measurement may be seen as just an interaction between the measured system and the measuring setup, and therefore a process to be studied within the theory itself.

In sharp contrast the measurement may give rise to a large perturbation in quantum physics. A consequence of this fact is that, whilst the simultaneous measurement of two observables on a macroscopic system perturb but slightly each other, the perturbation may be very relevant in joint measurements of microscopic systems. Therefore in classical theories we may assume that all observables of a system are compatible, in the sense that they may be measured simultaneously. (In macroscopic systems there are examples of obsevables that cannot be measured simultaneously, but they are scarce and irrelevant for the formulation of classical theories). In sharp contrast for microsopic systems there are incompatible observables that cannot be measured with the same experimental setup. The quantum mechanical formalism incorporates this fact in a fundamental form, namely two observables are compatible if and only if the associated operators commute.

\subsubsection{Incompatible observables}

The existence of incompatible measurements in microscopic systems leads logically to the possibility that the results of joint measurements of two (or several) observables depend on the context. For instance, the measurement of the position of a particle requires a different experimental context than the measurement of momentum. Therefore the joint measurement of position and spin requires a context different from the one for a joint measurement of momentum and spin. Thus we might expect that the spin measurement (in the same state, that is after the same preparation procedure) gives different results in the two cases. This is not so in our example (in fact the result for the spin measurement would the same in both cases) but different results are actually obtained in more complex examples, involving measurements 
of more than two observables. The property is a consequence of the quantum formalism and it is formally stated by the Kochen-Specker theorem (see below).

\subsubsection{Are there objective properties?}

As said above in classical physics it is assumed that measurements just reveal properties of the physical systems, existing with independence of observations. That is, in any actual (pure) state of a classical system all observable quantities have a well defined value. A mixed state (or statistical ensemble of systems) is associated to a joint probability distribution of the observables. This is not true in quantum physics, where it is not always possible to associate a joint probability distribution to all observables in a specific quantum state (i.e. to a given preparation procedure of the system). This fact has led some people to claim that, in contrast with classical physics, quantum systems have no properties whenever they are not measured. That is the observable properties "are created" or "emerge" as a result of measurements. This claim seemed bizarre to many people including Einstein, who criticized it with the rhetorical sentence: Is the moon there when nobody looks?.

In order to clarify the subject it is necessary to distinguish between the objective properties or "elements of reality" [22], that exist independently of any observation, and the "observable quantities" which appear only as a consequence of the observation or measurement. This is true in both classical and quantum physics. The point may be seen with an examples. In playing dice, every die has spots on the six faces with the numbers 1 to 6 . These are elements of reality, always present in the faces of the die. But if we play dice the value of our observable is only one number per die, namely the one printed in the upper face when the die becomes at rest on the table. The value of the observable, but not the objective property, is "created" by the action of playing dice, i. e. the experiment. In summary we should distinguish between objective properties and observables both in classical and quantum physics. However the connection between both is rather obvious in classical physics but less clear in quantum physics.

I conclude that the differences between classical and quantum theories do not provide sufficient support for the claim that objective properties (or elements of reality) do not exist in quantum systems or that realistic interpretations of quantum physics are not possible, that is interpretations in terms of "pictures of the reality" [22]. These pictures might be achieved by means 
of ontic or hidden variables models of quantum systems, to be studied in the next subsection.

\subsection{Ontic models in classical and quantum physics}

Following the advise of the initial paragraph of the EPR paper we may assume that a physical system is at any time in some "ontic state", that is "a real physical state not necessarily completely described by any theory (e. g. quantum), but objective and independent of the observer" 24]. The observable properties of the system derive from the ontic state at the time of observation 25$]$.

The standard classical assumption that measurements just reveal existing properties may be formalized stating that the observed result, $a$, depends on the ontic state, $\lambda$, of the system and the measuring set up, $A$, appropriate for a given observable quantity. That is the observed result, $a$, will be a function

$$
a=a(\lambda, A)
$$

At this moment I stress that this relation offers a "picture of the objective reality" as demanded by EPR, in the sense that it provides a causal connection between system plus measuring setup and the result obtained. In contrast the assumption that the results of the measurement "emerge" from the act of measurement would not provide any clear picture. This is true specially if it is assumed that the randomness of the result is "essential" in the sense that it is not caused by the incomplete control of all the parameters in the measurement. Eq.(16) explains the randomness of the result from the incomplete control of either the preparation procedure (leading to a statistical distribution of ontic states, $\lambda$ ) or the measuring setup, or both.

The assumption that the results of all observations on a system derive from functions like $a(\lambda, A)$, eq.(16) , would allow getting the joint probability of any set of observables, $\{A, B, \ldots C\}$. Without loss of generality we may consider that the set consists of observables with values $\{0,1\}$, because any observable may be defined in terms of yes-no questions. In this case any function like $a(\lambda, A)$ takes on the value 1 for some ontic states $\lambda$ and 0 for other states. Thus the joint probability distribution reduces to the knowledge of all expectations of products of observables, that is

$$
\langle A B \ldots C\rangle=\int f(\lambda) a(\lambda, A) b(\lambda, B) \ldots c(\lambda, C) d \lambda .
$$


Here we have assumed that $\lambda$ is a numerical parameter (or a set of parameters) attached to the ontic state so that the integral in $\lambda$ makes sense. The function $f(\lambda)$ gives the probability distribution of the ontic states in cases where we use a statistical ensemble of states (a "mixed state") due to incomplete information. For a pure state the function $f(\lambda)$ is zero except for some value of $\lambda$ (the function $f(\lambda)$ will have the form of a Dirac's delta) which reduces the right side of eq.(17) to a simple product (no integration is required).

The conclusion is that fixing the functions eqs.(16) for all possible observables of the system would determine their joint probability distribution. The reciprocal is also true. In fact, it is enough to identify the parameter $\lambda$ with the set of values of all observables.

The above construction, eq.(17), may be called "ontic model" 24] and extended, with some modifications, to quantum physics. For some people the appeal to ontic states is a philosophical (methaphysical) assumption that should not enter physics and prefer to treat $\lambda$ as a parameter in a model for an experiment and name it "hidden variable". Thus the construction may be called hidden variables model (HVM), which is the common name in the context of quantum physics.

Let us analyze whether HVM are possible in quantum mechanics. We consider a quantum system (pure or mixed), a state given by the density operator $\hat{\rho}$, and the set of projection operators $\{\hat{A}, \hat{B}, \ldots\}$. The projectors, fulfilling $\hat{A}^{2}=\hat{A}$, are the operators associated to dichotomic observables with values $\{0,1\}$. We may try to construct a HVM (or ontic model), as in the classical case, via a set of functions $\{a(\lambda, A), b(\lambda, B), \ldots\}$ such that an equation similar to eq.(17) holds true. At a difference with the classical case, quantum mechanics does not predict expectations for all products of observables (or more correctly, the expectations depend on the ordering of the operators), but only for products of "compatible" observables, that is those whose associated operators commute with each other. For the sake of clarity I will start with a simple example.

Let us consider a state $\hat{\rho}$ of a quantum system and a set of 3 observables $\{A, B, C\}$ each with values $\{0,1\}$, represented in quantum mechanics by the projection operators $\{\hat{A}, \hat{B}, \hat{C}\}$. We assume that $\hat{A}$ commutes with both $\hat{B}$ and $\hat{C}$, but $\hat{B}$ does not commute with $\hat{C}$. (A physical instance would consists of the observables position, momentum and spin of a particle, although here 
we consider for simplicity that the possible values of every observable are 0 and 1). In this case quantum mechanics predicts the following expectations, to be obtained via equations similar to eq.(??),

$$
\langle A\rangle,\langle B\rangle,\langle C\rangle,\langle A B\rangle,\langle A C\rangle
$$

but it does not predict the expectations (involving incompatible observables, which cannot be measured simultaneously)

$$
\langle B C\rangle,\langle A B C\rangle \text {. }
$$

We shall assume that commuting (noncommuting) operators correspond to compatible (incompatible) observables. Therefore the expectations $\langle A\rangle,\langle B\rangle,\langle A B\rangle$ may be measured simultaneously in a context, say 1, and the observables $\langle A\rangle,\langle C\rangle,\langle A C\rangle$ may be measured in another context, say 2 , but it is not possible to measure the five expectations eq.(18) in the same context (otherwise the observables $A, B, C$ would be compatible). It is trivial to get functions $\left\{a_{1}(\lambda, A), b_{1}(\lambda, B)\right\}$ giving the expectations $\langle A\rangle,\langle B\rangle,\langle A B\rangle$ via eqs.(17). This would provide a HVM (or ontic model) for the context 1. Similarly we may obtain another HVM for the context 2 via the functions $\left\{a_{2}(\lambda, A), c_{2}(\lambda, C)\right\}$. The two HVM toghether may be called a single $H V M$, that is noncontextual (contextual) if $a_{1}(\lambda, A)=a_{2}(\lambda, A)$ (if $a_{1}(\lambda, A) \neq$ $\left.a_{2}(\lambda, A)\right)$. The example shows that noncontextual are a particular kind of HVM. Studying whether they are possible for all pairs $\{\hat{\rho}, P\}$ of a state, $\hat{\rho}$, and a set of observables, $P$, of a physical system is the aim of the next subsection.

It may be realized that a noncontextual HVM corresponds to a complete random variables representation of the pair $\{\hat{\rho}, P\}$, as defined in the mathematical section 2. Indeed the knowledge of all expectations like eqs.(17) determines a joint probability distribution of all observables. I stress that

the commutativity of all operators with each other is a sufficient condition for the existence of a noncontextual HVM, but it is not necessary.

\subsection{Contextual hidden variables. Kochen-Specker the- orem}

In a Hilbert space of dimension 3 or more, noncontextual hidden variables theories are not possible in general. 
The result is called Kochen-Specker theorem [26] for the authors who proved it in 1967, although it had been proved independently in 1966 by Bell[27], [28]. (It may be shown that for spaces of dimension 2 noncontextual HVM are always possible). The original Kochen-Specker proof will be given here, but once we know that noncontextual HVM correspond to complete random variables representations, a simple proof consists of finding an example violating a Bell inequality. Such examples are given by eqs.(12) or (14). In fact in that case it is not possible a complete random variables representation, and therefore a noncontextual HVM. The proof of the Kochen-

Specker theorem via Bell inequalities, here presented, has the advantage of suggesting empirical tests. Indeed experiments have been performed showing the violation of noncontextual HVM. In particular empirical Bell tests refute noncontextual HVM (and even the particular class of noncontextual that are also local HVM if the experiments block the locality loophole).

What makes necessary the use of contextual HVM is the fact that the operators associated to observables in quantum theory do not always commute with each other. But I shall point out that sometimes a noncontextual HVM is possible for systems where not all such operators commute. Indeed the possibility of noncontextual HVM is not a property of the system but it depends also on the quantum state. Thus "noncontextual" does not imply that the joint probability distribution of all observables may be measured in the same context. Indeed, for some states, noncontextual HVM are possible even if not all operators commute.

Noncontextual ontic models are always possible in classical physics, whence the impossibility of such models is a signal of quantum behaviour. Thus the Bell inequalities may be used, and have been used, in order to discriminate quantum vs. classical phenomena. If a Bell inequality is violated the phenomenon cannot be interpreted via noncontextual HVM and it should be considered specifically quantum.

\subsection{Bell's theorem}

Both in classical mechanics and in ordinary life the correlations between distant systems are assumed to derive from a common past. For instance the similarity between twins (possibly living in different cities) is a correlation between distant bodies. It is an obvious consequence of the common origin, 
which might be formalized stating

$$
\langle A B\rangle=\int f(\lambda) a(\lambda, A) b(\lambda, B) d \lambda
$$

where $A$ and $B$ label some feature of the twins, e. g. colour of the eyes, and $f(\lambda)$ represents the (common) genetic code. But correlations cannot be interpreted in terms of eqs.(19) or (17) whenever a Bell inequality is violated. Hence a fundamental consequence of Bell's work is to show that in nature there might be correlations between distant bodies not deriving from a common past; this would be the case if the correlations violated a Bell inequality. The result is a consequence of the laws of (standard) probabilities and it is therefore independent of the existence of quantum mechanics.

Quantum mechanics enters because it predicts the existence of such correlations, a result known as "Bell's theorem". Bell introduced hidden variables models, called local, that are partially contextual. He stressed that contextual HVM are not too strange provided contextuality means influence of the context (e. g. the measuring equipment) near the system under study. However it would be rather strange if the influence is at a distance, as in the experiments of the EPR type[22]. In those experiments two particles are produced in a source, each particle travels in a different direction and when they are far appart two different agents, Alice and Bob, measure their respective properties. In this case it is unplausible to assume that there may be an influence of the context of Alice (Bob) on the result of the measurement made by Bob (Alice). But those influences should exist if a Bell inequality is violated by the four expectations $\left\langle A_{1} B_{1}\right\rangle,\left\langle A_{1} B_{2}\right\rangle,\left\langle A_{2} B_{1}\right\rangle,\left\langle A_{2} B_{2}\right\rangle$, where $A_{j}\left(B_{k}\right)$ are observables measured by Alice (Bob).

It may be realized that the relevance of Bell's theorem is greater than just to refute a class of hidden variables theories of quantum mechanics. It proves that local realistic models of natural phenomena are not compatible with quantum mechanics. Indeed Bell himself reinterpreted the correlation between two measurements, one by Alice the other one by Bob within relativity theory[2]. To do that he considered the set of variables $\lambda$ to be the union of two sets, $\lambda_{a}$ and $\lambda_{b}$, consisting each of all events in the past light cone of the measurement performed by Alice and Bob, respectively. Therefore the violation of a Bell inequality with space-like separated measurements, in the sense of relativity theory, would imply that the correlation does not derive from the intersection of the said past light cones. 
Thus Bell's theorem seems to prove the incompatibility of quantum mechanics with relativity theory. The contradiction looks dramatic, but most authors think that there is no real contradiction because quantum mechanics does not allow sending superluminal signals from Alice to Bob (or from Bob to Alice). Actually for many authors what is proved by Bell's theorem is the nonexistence of hidden variables or maybe the unavoidability of an instrumentalistic interpretation of quantum mechanics, excluding realistic interpretations. For this reason it is frequent to state Bell's theorem as proving the incompatibility between local realism and quantum mechanics.

In view of Bell's theorem many experiments have been performed during the latter forty years in order to test empirically local realism against quantum mechanics. The experiments confirmed quantum mehcanics but, due to the existence of loopholes, they were unable to refute local realism. Only recently has been possible to perform loophole-free experiments [3], [4], [5].

\subsection{Entlanglement, the characteristic trait of quantum mechanics}

One of the main difficulties for a realistic understanding of the quantum formalism derives from the phenomenon of entanglement. Known since the early days of quantum mechanics it was crucial in the EPR argument mentioned above 22]. Its relevance was emphasized by Schrödinger in a celebrated paper [29]. He stated that entanglement "is not one but the characteristic trait of quantum mechanics". The problem is that getting a clear physical picture of entanglement is difficult. In fact, its standard definition is not made in terms of physical concepts, but requires abstract mathematics: "Several physical systems are entangled if their joint wavefunction (or statevector) cannot be written as the product of wavefunctions, one for each system". As a consequence the common wisdom is that entanglement cannot be pictured in terms of physical models. Actually a physical interpretation has been proposed that considers entanglement as a correlation between the fluctuations of the vacuum fields acting on different systems (maybe separated at a long distance) [25]. However this interpretation is not popular.

One of the difficulties put by the phenomenon of entanglement may be seen with the following argument. Let us consider an entangled (singlet) state of two spin-1/2 particles. It may be represented (ignoring normalization) in 
the form

$$
\Psi=(\uparrow \downarrow-\downarrow \uparrow)
$$

which may be expressed in words saying that either electron \#1 has spin up and electron \#2 spin down or electron \#1 has spin down and electron \#2 spin up. The strange fact is that the state eq.(20) is pure, which is interpreted as a complete knowledge of the whole system, and nevertheless we have no information about the spin of every electron. We only know that there are 50-50\% probabilities for the two possibilities, up and dawn, and in fact these would be the probabilities of the results if measurements were performed. That is, according to the standard quantum view we have complete information about the whole two-spin system but no information about every part. (It is like a student claiming to have complete knowledge of a whole subject matter but no knowledge at all about every lesson). This is paradoxical because in ordinary life complete information just means information about every part. This statement is true not only in classical physics but in all sciences where standard (Kolmogorov) probability theory is used, e.g. in economics or biology. The fact may be put in quantitative form in terms of "entropy", a measure of our ignorance (our lack of information). In fact in a system consisting of several parts the classical (Shannon) entropy fulfils the inequalities

$$
S_{j} \leq S \leq \sum_{j} S_{j}
$$

where $S_{j}$ is the entropy of subsystem $j$ and $S$ the entropy of the whole system. The latter inequality is called subadditivity and it is not too relevant here. The former means that our lack of information about a system cannot be smaller than about one of the parts. This is violated in quantum theory, where the standard measure of ignorance is von Neumann entropy [30], defined by

$$
S=-\operatorname{Tr}(\hat{\rho} \log \hat{\rho}), S_{j}=-\operatorname{Tr}\left(\hat{\rho}_{j} \log \hat{\rho}_{j}\right) .
$$

In fact for the state eq.(20) the von Neumann entropy fulfils

$$
S=0, S_{1}=S_{2}=\log 2 .
$$

It is possible to show that the violation of the information inequality, former eq.(21), is closely related to the violation of a Bell inequality [32]. Indeed a necessary condition for both is that the quantum state is entangled. 
A naive solution to the information problem would be assuming that the quantum statevector eq.(20) represents a statistical mixture (of the two possibilities $\uparrow \downarrow$ and $\downarrow \uparrow$ ). However this assumption poses well known difficulties because in quantum physics a linear combination like eq.(20) has empirically testable differences with a mixture. Of course in hidden variables models (possibly contextual) the von Neumann entropy eq.(22) is no longer the appropriate measure of information.

Entanglement is quite common in quantum physics. For instance in many-electron systems (atoms, molecules and solid bodies) the Pauli principle gives rise to entanglement. The fact could be illustrated with the statevector of a two-electron atom. If the total spin is zero (ignoring nuclear spin) the joint statevector might be written

$$
\Psi(1,2)=\psi\left(\mathbf{r}_{1}, \mathbf{r}_{2}\right)(\uparrow \downarrow-\downarrow \uparrow),
$$

where $\psi\left(\mathbf{r}_{1}, \mathbf{r}_{2}\right)$ usually does not have the form of a product of one-electron wavefunctions. In this case eq.(23) represents an entangled state involving position and spin. However entanglement is most relevant when the systems are separated, as stressed in the EPR paper [22].

We see that entanglement is a correlation, but a strange correlation different from the classical one by the fact that it may violate a Bell inequality. Indeed the close connection of entanglement with the Bell inequalities derives from the fact that entanglement is, in addition to necessary, a sufficient condition for the existence of a Bell inequality violated by (ideal) quantum predictions [31.

An interesting question related to entanglement appears if quantum mechanics is universally valid, which is the common wisdom today. (Thus classical theories are seen as approximations to quantum theories, the passage from quantum to classical being an active subject of research in recent years). If quantum mechanics is universally valid and we accept the unrestricted validity of the superposition principle then all statevectors correspond to physical states of the system. This assumption leads to bizarre consequences in the macroscopic domain as was emphasized by Schrödinger [29] with his celebrated cat example. It consists of a cat plus a radiactive atom such that the cat is killed when the atom decays. If every vector of the Hilbert space corresponds to a state of the system, then there exists amongst others a state represented by the linear combination

$$
\left.\left.\frac{1}{\sqrt{2}}(\mid \text { livecat }, 1\rangle+\mid \text { deadcat }, 0\right\rangle\right),
$$


where the first term represents the cat alive with the atom excited and the second term the cat dead with the atom in the ground state.

It is obvious that the description of the state of a macroscopic system cannot be made in practice associating to it a statevector and that a density operator is a more appropriate description. The usual solution to the difficulty is to take into account that a cat, as any macroscopic system, cannot be isolated from the environment. Therefore the state of the cat should not be represented by a pure state like eq.(??), but by a reduced density operator resulting from taking the partial trace over the degrees of freedom of the environment. The reduced density operator might be interpreted as incomplete information, with some proability $P$ that the cat is alive and the probability $1-P$ that she is dead. This approach to macroscopic quantum systems is named decoherence induced by the environment, and it leads from a pure state of a large system to a reduced density operator for any subsystem. The density operator is named an improper mixture, meaning that it is different from the mixture corresponding to an isolated sysem prepared in a state without complete control of all relevant parameters (complete control would lead to a pure state). However decoherence theory just translates the problem from the cat to the universe. Indeed it is difficult to stablish a rigorous spatial limit to the environment and if no limit is put we should consider the whole universe. Thus the decoherence approach is closely connected to the many worlds interpretation of quantum mechanics 33. In this case we should assume that, after the evolution of the cat interacting with the environment, the universe would consist of a linear combination of statevectors everyone with a copy of the atom, the cat and everything else including "me" (the observer). For many people a linear combination of two terms, one of them with "me" seing a dead cat and another one with "me" seing an alive cat is even more bizarre than a linear combination of living and dead cat. In spite of this the "wavefuction of the universe" has been advocated by respected physicists.

The Schrödinger cat example is also relevant for the discussion of measurement in quantum theory. In fact a measurement amounts to an interaction of a system (usually small) with a measuring device (usually macroscopic). If the state of system plus device is represented by a wavefunction, then after the interaction the composite system will be entangled. Indeed in the Schrödinger example the cat may be taken as a measuring device for the state of the atom. The measurement problem has been crucial for the interpretation of quantum mechanics, but a more detailed discussion is beyond 
the scope of this paper (see e.g. [25]).

\section{References}

[1] J. S. Bell, On the Einstein Podolsky Rosen paradox. Physics, 1, 195-200 (1964) (Reprinted in Bell (1987)).

[2] J. S. Bell, Speakable and unspeakable in quantum mechanics. Cambridge University Press, Cambridge, 1987. This book contains reprints of most Bell's papers about foundations of quantum mechanics.

[3] B. Hensen et al., Experimental loophole-free violation of a Bell inequality using entangled electron spins separated by $1.3 \mathrm{~km}$, doi.org/10.1038/nature 15759 (2015); arXiv: 1508.05949.

[4] L. K. Shalm et al., A strong loophole-free test of local realism, Phys. Rev. Lett. 115, 250402 (2015); arXiv: 1511.03189.

[5] M. Giustina et al., A significant loophole-free test of Bell's theorem with entangled photons, Phys. Rev. Lett. 115, 250401 (2015); arXiv: 1511.03190 .

[6] N. Brunner, D. Cavalcanti, S. Pironio, V. Scarani and S. Wehner, Bell nonlocality. Rev. Mod. Phys. 86, 419478 (2014).

[7] C. A. Hooker (ed.), The Logico-algebraic Approach to Quantum Mechanics, Vols. I and II, Reidel, Dordrecht. 1975, 1979.

[8] E. Santos, The Bell inequalities as tests of classical logic. Phys. Lett. A 115, 363-365 (1986).

[9] Itamar Pitovsky, Quantum Probability-Quantum Logic. Springer, Berlin. 1989.

[10] E. Beltrametti and M. Maczyński, Problem of classical and nonclassical probabilities", Int. J. Th. Phys. 31, 1849 - 1856 (1992).

[11] A. Dvurecenskij and H. Langer, Bell-type inequalities in orthomodular lattices, I, II, Int. J. Th. Phys. 34, 995 - 1024 and 1025 - 1036 (1995). 
[12] S. Pulmannova, Hidden variables and Bell inequalities on quantum logics, Found. Phys. 32, 193 - 216 (2002).

[13] J. Pykacz, J. Valaskova and O. Nanasiova, Bell-type inequalitites for bivariate maps on orthomodular lattices, Found. Phys. 45 $900-913$ (2015).

[14] G. Auletta, Foundations and interpretation of quantum mechanics. World Scientific, Singapore. 2000.

[15] F. Laloë, Do we really understand quantum mechanics?. Strange correlations, paradoxes, and theorems. Cambridge University Press. 2012.

[16] M. Redhead, Incompleteness, nonlocality and realism. Clarendon Press. Oxford. 1990.

[17] B. d'Espagnat, Veiled reality. An analysis of present-day quantum mechanical concepts. Westview Press. 2003.

[18] B. d'Espagnat, On physics and philosophy. Princeton University Press. 2006 .

[19] B. G. Englert, On quantum theory. Eur. Phys. J. D 67, 238 (2013), 16 pages.

[20] F. J. Clauser and M. A. Horne, Experimental consequences of objective local theories. Phys. Rev. D 10, 526-535 (1974).

[21] F. J. Clauser, M. A. Horne, A. Shimony and R. A. Holt, Proposed experiment to test local hidden-variables theories, Phys. Rev. Lett. 23, 880-883 (1969).

[22] A. Einstein, B. Podolsky and N. Rosen, Can quantum-mechanical description of physical reality be considered complete?, Phys. Rev. 47, 777-780 (1935).

[23] N. Bohr, Can quantum-mechanical description of physical reality be considered complete?, Phys. Rev. 48, 696-670 (1935).

[24] N. Harrigan and R. W. Spekkens, Einstein, incompleteness, and the epistemic view of quantum states, Found. Phys. 40, 125-157 (2010). 
[25] E. Santos, Towards a realistic interpretation of quantum mechanics providing a model of the physical world, Foundations of Science, DOI 10.1007/s10699-014-9366-y (2015).

[26] S. Kochen and E. Specker, The problem of hidden variables in quantum mechanics, J. Math. Mech. 17, 59-87 (1967).

[27] J. S. Bell, On the problem of hidden variables in quantum mechanics. Rev. Mod. Phys. 38, 447-452 (1966). (Reprinted in Bell (1987)).

[28] D. N. Mermin, Hidden variables and the two theorems of John Bell, Rev. Mod. Phys. 65, 803-815 (1993).

[29] E. Schrödinger, The present situation of quantum mechanics. Naturwissenschaften, 23: pp. 807-812; 823-828; 844-849 (1935).

[30] J. von Neumann, Mathematishe Grundlagen der Quantenmechanik. Berlin: Springer-Verlag. 1932. English translation Princeton University Press, 1955.

[31] N. Gisin, Bell inequality holds for al non-product states, Phys. Lett. A 154, 201 (1991).

[32] E. Santos, Entropy inequalities and Bell inequalities for two-qubit systems, Phys. Rev. A 69, 022305-1 to 6 (2004); A 70, 1 (E) (2004).

[33] B. S. de Witt and N. Graham, The many-worlds interpretation of quantum mechanics. Princeton University Press. 1973. 